\title{
Association of diet quality with dietary inflammatory potential in youth
}

\author{
Rowaedh Ahmed Bawaked ${ }^{\mathrm{a}, \mathrm{b}}$, Helmut Schröder ${ }^{\mathrm{a}, \mathrm{c}}$, Lourdes Ribas-Barba ${ }^{\mathrm{d}, \mathrm{e}}$, Maria Izquierdo-Pulido ${ }^{\mathrm{e}, \mathrm{f}}$, \\ Carmen Pérez-Rodrigo ${ }^{g}$, Montserrat Fíto ${ }^{\mathrm{a}, \mathrm{e}}$ and Lluis Serra-Majem ${ }^{\mathrm{e}, \mathrm{h}}$
}

${ }^{a}$ Cardiovascular Risk and Nutrition Research Group (CARIN), IMIM (Hospital del Mar Medical Research Institute), Barcelona, Spain; bepartment of Experimental and Health Sciences, University of Pompeu Fabra, Barcelona, Spain; 'C CIBER Epidemiology and Public Health (CIBERESP), Instituto de Salud Carlos III, Madrid, Spain; ' $F$ Fundación para la Investigación Nutricional (Nutrition Research Foundation), Barcelona, Spain; eCIBER Physiopathology of Obesity and Nutrition (CIBEROBN), Instituto de Salud Carlos III, Madrid, Spain; fDepartment of Nutrition, Food Sciences and Gastronomy, University of Barcelona, Barcelona, Spain; 9FIDEC Foundation, University of the Basque Country (UPV/EHU)Bilbao, Bilbao, Spain; hesearch Institute of Biomedical and Health Sciences, University of Las Palmas de Gran Canaria, Las Palmas, Spain

\begin{abstract}
Background: Diet plays a crucial role in the regulation of chronic inflammation. The sparse evidence available in adult populations indicates that diet quality is linked to the dietary inflammatory potential; however, this association has not been established in youth.

Design: Data were obtained from a representative national sample of 2889 children and young people in Spain, aged 6-24 years. The dietary inflammatory potential was measured by the dietary inflammatory index (DII), and diet quality by three conceptually different measures: the Mediterranean Diet Quality Index for children and adolescents (KIDMED), energy density, and total dietary antioxidants capacity.

Results: The mean DII was $1.96 \pm 0.76$ units Scoring for the KIDMED index and the total dietary antioxidant capacity significantly decreased ( $p<0.001$ and $p=0.030$, respectively) across quintiles of the DII, whereas the opposite was true for energy density $(p<0.001)$. The effect size of these associations was strongest for energy density, followed by the KIDMED index and total dietary antioxidant capacity.

Conclusion: A healthy diet characterized by high adherence to the Mediterranean diet, high total dietary antioxidant capacity, or low energy density was linked to greater anti-inflammatory potential of the diet, as measured by the DII.
\end{abstract}

ARTICLE HISTORY

Received 6 February 2017

Accepted 8 May 2017

\section{KEYWORDS}

Dietary inflammatory index; KIDMED; energy density; total dietary antioxidant; children; adolescents; enKid

\section{Introduction}

Diet plays a crucial role in the regulation of chronic inflammation $[1,2]$. The Western dietary pattern, high in refined grains, sugars, simple carbohydrates, red meat, and high-fat dairy products, increases the levels of pro-inflammatory markers such as C-reactive protein (CRP) and interleukin-6 (IL-6) [1,3]. In contrast, a traditional Mediterranean diet with generous consumption of fruits, vegetables, whole grains, legumes, increased consumption of fish and nuts, and higher use of olive oil in food preparation is associated with lower levels of pro-inflammatory biomarkers, including endothelial adhesion molecules, CRP, and tumor necrosis- $\alpha$ (TNF- $\alpha$ ) [2]. Evidence shows that several chronic diseases, including cardiovascular diseases and cancer, are triggered in part by inflammation $[4,5]$, and atherosclerosis progresses from childhood and adolescence to adulthood [6].
A high score on the dietary inflammatory index (DII), which was developed to assess the inflammatory potential of the diet [7], has been associated with inflammation biomarkers such as CRP [8,9], IL-6 [10,11], homocysteine [10], and TNF- $\alpha$ [11]. In addition, an increased DII score has been associated with cancer [12], asthma [13], and cardiovascular diseases [14].

Limited evidence has positively associated diet quality with the dietary anti-inflammatory potential in adult populations [15-17], but data for children and young people are missing. Furthermore, there is no consensus about the definition of diet quality [18]. Indeed, scientists have proposed many different measures of diet quality [18]. In this study, we hypothesized that high anti-inflammatory potential is characteristic of three conceptually different measures of high diet quality in youth: adherence to the Mediterranean diet, total dietary antioxidant potential, and energy density.

CONTACT Helmut Schröder HSchroeder@imim.es Cardiovascular Risk and Nutrition Research Group (CARIN), IMIM (Hospital del Mar Medical Research Institute), Barcelona, Spain.

(-) Supplemental data for this article can be accessed here.

( 2017 The Author(s). Published by Informa UK Limited, trading as Taylor \& Francis Group

This is an Open Access article distributed under the terms of the Creative Commons Attribution License (http://creativecommons.org/licenses/by/4.0/), which permits unrestricted use, distribution, and reproduction in any medium, provided the original work is properly cited. 
The objective of this study was to determine dietary inflammatory potential, measured by the DII, and its association with diet quality indicators in a representative sample of Spanish youth.

\section{Methods}

\section{Study design}

The enKid Study was a cross-sectional survey of the nutritional status and food habits of 3534 Spanish children and young adults, conducted between 1998 and 2000. Participants were selected by multistage random sampling procedures based on a population register. The objective of the enKid Study, described in detail elsewhere [19], was two-fold: (I) to establish the prevalence of micronutrient deficiencies in the population aged 2-24 years; and (II) to analyze the association of these micronutrients with gender and age groups.

The sample size was calculated according to (i) the estimated prevalence of most micronutrients with 95\% confidence interval and an accuracy of $\pm 2.5 \%$ of the average value of the micronutrient; and (ii) a statistical power of $80 \%$ to detect significant differences between two groups $=10 \%$ of the mean of the micronutrients (setting the alpha error at $p=0.05$ ). The calculated sample size of 3850 individuals was overestimated by $30 \%$, resulting in a theoretical sample size of 5500 individuals. The final sample size of the enKid Study was 3534 individuals (Supplementary Figure 1). We excluded 385 children aged 2-5 years to concentrate the study population in a narrower age range. Individuals with incomplete dietary data were also excluded $(n=250)$. The final sample consisted of 2889 individuals aged 6-24 years.

Parental written consent was obtained on behalf of each participant younger than 18 years. The study protocol was approved by the ethics committee of the Spanish Society of Community Nutrition.

\section{Dietary data collection}

Dietary data were collected during in-home interviews carried out by 43 trained dietitians and nutritionists using household measures to estimate portion sizes. Dietary intake was assessed by means of a $24 \mathrm{~h}$ recall. A second $24 \mathrm{~h}$ recall was completed in a random sample of $25 \%$ of the participants on an independent non-consecutive day. The administration of the second questionnaire allowed for the adjustment of intakes for random intra-individual variation using the method described by Liu et al. [20]. The same field staff entered survey data into software specifically designed for the study.

\section{Dietary inflammatory index (DII)}

The inflammatory properties of each participant's diet were assessed from the $24 \mathrm{~h}$ recall data using the DII, which is based on a review and analysis of 1939 scientific articles [7]. These articles studied the relationship between 45 dietary components and six inflammatory markers (CRP, IL- $1 \beta$, IL-4, IL-6, IL-10, and TNF- $\alpha$ ) derived from cell culture and animal experiments, and from cross-sectional, longitudinal, and intervention trials in humans. Each food parameter in each article was scored by assigning $(+1)$ for pro-inflammatory effect, $(-1)$ for anti-inflammatory effect, or (0) for no effect, and weighted according to the study design. In the present study, 23 of the 45 DII food parameters were available (fiber, protein, carbohydrates, cholesterol, total fat, trans fat, saturated fat, monounsaturated fat, polyunsaturated fatty acids, omega- 3 and omega- 6 fatty acids, iron, magnesium, energy intake, and vitamins $A, B_{1}$, $\mathrm{B}_{2}, \mathrm{~B}_{5}, \mathrm{~B}_{6}, \mathrm{~B}_{12}, \mathrm{C}, \mathrm{D}$, and $\mathrm{E}$ ). For each participant, each food parameter intake score was subtracted from the mean of 11 countries from around the world and divided by its standard deviation. $Z$ scores and centered percentiles were calculated to reduce the effect of right skewing. For each food parameter, the centered percentile was multiplied by the overall inflammatory effect. All DII scores for the food parameters were then summed to create the overall DII score for each participant, which ranged from -6.77 , representing maximum anti-inflammatory properties, to 7.79 , representing maximum pro-inflammatory properties. The development and validation of the DII have been explained in greater detail elsewhere [7].

\section{Diet quality measures}

Energy density and total dietary antioxidant capacity were calculated from the $24 \mathrm{~h}$ recalls. The Mediterranean Diet Quality Index for children and adolescents (KIDMED) index was based on a 16-item questionnaire administered separately from the recalls as part of the enKid Study $[21,22]$.

\section{Energy density}

There is no consensus about the best method to measure dietary energy density [23]. To allow comparability with other studies, we present data based on dietary density calculations that included food only [energy intake (kcal) from all foods consumed divided by the corresponding weight ( $\mathrm{g}$ ) of the foods] and all foods together with all caloric beverages [energy intake (kcal) from all foods and caloric beverages consumed divided by the corresponding weight ( $\mathrm{g}$ ) of the foods and beverages]. 


\section{Total dietary antioxidant capacity}

Dietary total antioxidant capacity was estimated using published data of antioxidant capacity in foods measured by ferric-reducing antioxidant power (FRAP) assays [24].

\section{KIDMED index}

The KIDMED index estimates adherence to the Mediterranean diet in children and young adults, considering the principles that sustain Mediterranean dietary patterns and those that undermine it. Items denoting lower adherence were assigned a value of -1 (four items) and those related to higher adherence were scored $+1(12$ items). Scores ranged from -4 to 12 , with higher scores indicating greater adherence to the Mediterranean diet and scores below 6 points defined as low Mediterranean diet adherence.

\section{Covariates}

Data on physical activity and maternal education were reported by participants responding to an intervieweradministered questionnaire. The physical activity questionnaire included 14 questions on type and frequency of physical activity and time spent at school and outside school doing physical activity. Maternal education level was recorded as: (i) no education (never went to school); (ii) primary education not completed; (iii) completed primary education; (iv) secondary education; and (v) university. Basal metabolic rate (BMR) was estimated with Schofield equations based on gender, age, weight, and height [25]. Implausible reporters of energy intake were identified by replacing Goldberg's single cut-off [26] with age- and gender-specific cut-off values. These values consisted of the $95 \%$ confidence limits of agreement between physical activity level (PAL) and the ratio of energy intake to BMR. The following formula was used:

$$
\text { Cut }- \text { off }=\mathrm{PAL} \times \exp \left[ \pm 1.96 \times \frac{(S / 100)}{\sqrt{n}}\right]
$$

where

$$
S=\sqrt{\left[\frac{\left.C V_{\mathrm{wEI}}^{2}+C V_{\mathrm{wBMR}}^{2}+C V_{\mathrm{tP}}^{2}\right]}{d}\right.}
$$

Intra-individual variations in energy intake $\left(\mathrm{CV}^{2} \mathrm{wEI}\right)$ and $\mathrm{BMR}\left(\mathrm{CV}^{2} \mathrm{wBMR}\right)$ and inter-individual variation in PAL $\left(\mathrm{CV}^{2} w \mathrm{Pt}\right)$ were calculated using gender- and agespecific reference values [27-29]. The single Goldberg PAL of 1.55 was replaced by gender- and age-dependent PAL. We estimated dietary intake by one $24 \mathrm{~h}$ recall and set the number of days to 1 .

\section{Statistical analysis}

General linear modeling procedures were used to compare baseline participant characteristics by quintiles of DII. Polynomial contrast was used to determine overall $p$ for linear trend for continuous variables with normal distribution, and the Kruskal-Wallis test to determine overall $p$ for non-normal distributions. The $p$ for linear trend for categorical variables was obtained by the Mantel-Haenszel linear-by-linear association chi-square test.

To determine the association between DII and diet quality, we fitted general linear models adjusted for gender, age, energy intake, energy underreporting, region, community size, and maternal education level. $Z$ scores of energy density, total dietary antioxidant capacity, and the KIDMED index were created to compare the effect size of the associations with the DII.

Calculation of Cohen's $\kappa$ was based on the observed versus the expected agreement and used to test the strength of agreement between the tertile distribution of energy density, total dietary antioxidant capacity, and the KIDMED index. To explore effect modification due to DII and energy overreporting and underreporting, we modeled interaction terms for DII/energy overreporting and DII/energy underreporting. Associations were considered significant if $p<0.05$. SPSS for Windows version 18 (SPSS, Chicago, IL, USA) was used for all statistical analyses.

\section{Results}

The mean DII was $1.25 \pm 1.39$ units, with a range of -4.27 to 4.16 (Table 1). Participants with high DII scores were more likely to be female, were younger, and had fewer total physical activity minutes per day. The proportion of participants with a high DII was greater in communities of 10,000-50,000 inhabitants and lower in communities of more than 350,000 inhabitants (Table 1).

Adherence to the Mediterranean diet, total dietary antioxidant capacity, intake of protein, polyunsaturated fatty acids, fiber, magnesium, vitamins $\mathrm{C}, \mathrm{E}, \mathrm{B}_{6}, \mathrm{~B}_{2}$, and $B_{1}$, and intake of fruits, vegetables, legumes, and fish decreased across the DII. The opposite was true for dietary energy density and intake of lipids, monounsaturated fat, saturated fat, calcium, dairy, cereals, meat, pastry, and cakes and sweets. Higher DII was associated with a decreased proportion of energy underreporting and increased overreporting (Table 2).

General linear modeling procedures adjusted for age, gender, energy consumption, energy underreporting, community size, region, and maternal education level revealed a negative association between DII, KIDMED index score, and total dietary antioxidant capacity (Table 3). In 
Table 1. General characteristics across quintiles (Q1-Q5) of the dietary inflammatory index (DII).

\begin{tabular}{|c|c|c|c|c|c|c|}
\hline & $\begin{array}{c}\text { Q1 } \\
(n=578)\end{array}$ & $\begin{array}{c}\text { Q2 } \\
(n=579)\end{array}$ & $\begin{array}{c}\mathrm{Q} 3 \\
(n=577)\end{array}$ & $\begin{array}{c}\mathrm{Q} 4 \\
(n=578)\end{array}$ & $\begin{array}{c}\mathrm{Q} 5 \\
(n=577)\end{array}$ & $\boldsymbol{p}^{\mathrm{a}}$ \\
\hline DII & $0.79(-1.17 ; 1.35)$ & $1.63(1.35 ; 1.87)$ & $2.06(1.87 ; 2.26)$ & $2.42(2.26 ; 2.61)$ & $2.87(2.61 ; 3.66)$ & $<0.001$ \\
\hline Male, $n(\%)$ & $336(58.1)$ & $291(50.3)$ & $242(41.9)$ & $229(39.6)$ & $220(38.1)$ & $<0.001$ \\
\hline Age (years) & $19.2(18.8,19.6)$ & $17.5(17.1,17.9)$ & $16.9(16.5,17.3)$ & $15.7(15.3,16.1)$ & $14.5(14.1,14.9)$ & $<0.001$ \\
\hline Physical activity (min/day) & $145(134,156)$ & $134(123,145)$ & $127(116,138)$ & $130(119,141)$ & $127(116,138)$ & 0.024 \\
\hline Maternal education, $n(\%)^{\mathrm{b}}$ & $112(19.6)$ & 99 (17.4) & $93(16.3)$ & $108(18.8)$ & $87(15.2)$ & 0.460 \\
\hline Community size, $n(\%)^{c}$ & & & & & & $<0.001$ \\
\hline$<10,000$ & $130(22.5)$ & $119(20.6)$ & $132(22.9)$ & $126(21.8)$ & $126(21.8)$ & \\
\hline $10,000-50,000$ & $104(18.0)$ & $130(22.5)$ & $140(24.3)$ & $168(29.1)$ & $177(30.7)$ & \\
\hline $50,000-350,000$ & $150(26.0)$ & $158(27.3)$ & $150(26.0)$ & $175(30.3)$ & $163(28.2)$ & \\
\hline$>350,000$ & $194(33.6)$ & $172(29.7)$ & $155(26.9)$ & 109 (18.9) & $111(19.2)$ & \\
\hline Region, $n(\%)^{\mathrm{d}}$ & & & & & & 0.837 \\
\hline Central & $144(24.9)$ & $146(25.2)$ & $141(24.4)$ & $155(26.8)$ & $133(23.1)$ & \\
\hline Northeast & $151(26.1)$ & $137(23.7)$ & 134 (23.2.6) & $140(24.2)$ & $130(22.5)$ & \\
\hline North & $120(20.8)$ & 112 (19.3) & $142(24.6)$ & $128(22.1)$ & $142(24.6)$ & \\
\hline South & 75 (13.0) & $93(16.1)$ & $76(13.2)$ & 77 (13.3) & $83(14.4)$ & \\
\hline East & $71(12.3)$ & 73 (12.6) & $64(11.1)$ & $63(10.9)$ & $70(12.1)$ & \\
\hline Canary Islands & $17(2.94)$ & $18(3.11)$ & $20(3.47)$ & $15(2.6)$ & $19(3.29)$ & \\
\hline
\end{tabular}

Data are shown as mean and range for DIl; mean and 95\% confidence interval for continuous variables (age and physical activity); and proportions within quintiles for categorical variables (male gender, maternal education level, community size, and region).

${ }^{a} p$ Values were obtained by ANOVA, Kruskal-Wallis, and Pearson chi-square for normal continuous, non-normal continuous, and categorical variables, respectively.

${ }^{\mathrm{b}}$ Maternal education expressed as proportion with university degree.

'Percentage expressed as proportion within community size.

dPercentage expressed as proportion within region.

Table 2. Diet quality across quintiles (Q1-Q5) of the dietary inflammatory index (DII).

\begin{tabular}{|c|c|c|c|c|c|c|}
\hline Characteristic & $\begin{array}{c}\text { Q1 } \\
(\mathrm{n}=578)\end{array}$ & $\begin{array}{c}\text { Q2 } \\
(\mathrm{n}=579)\end{array}$ & $\begin{array}{c}\text { Q3 } \\
(\mathrm{n}=577)\end{array}$ & $\begin{array}{c}\text { Q4 } \\
(\mathrm{n}=578)\end{array}$ & $\begin{array}{c}\text { Q5 } \\
(n=577)\end{array}$ & $\boldsymbol{p}^{\mathrm{a}}$ \\
\hline KIDMED index & $7.7(7.5,7.8)$ & $7.2(7.1,7.4)$ & $7.0(6.8,7.1)$ & $6.9(6.7,7.1)$ & $6.6(6.5,6.8)$ & $<0.001$ \\
\hline Dietary antioxidant capacity & $11.4(10.7,12.1)$ & $9.8(9.0,10.5)$ & $9.0(8.2,9.7)$ & $8.7(7.9,9.4)$ & $7.6(6.9,8.4)$ & $<0.001$ \\
\hline Dietary energy density & $0.93(0.91,0.96)$ & $1.02(0.99,1.04)$ & $1.06(1.03,1.09)$ & $1.12(1.09,1.15)$ & $1.2(1.18,1.23)$ & $<0.001$ \\
\hline Energy intake (kcal/day) & $2272(2239,2304)$ & $2073(2040,2105)$ & $1974(1941,2007)$ & $1927(1895,1960)$ & $1951(1919,1984)$ & $<0.001$ \\
\hline Energy underreporting, $n(\%)$ & $97(16.8)$ & $118(20.4)$ & $113(19.7)$ & $114(19.7)$ & $81(14.1)$ & 0.030 \\
\hline Energy overreporting, $n(\%)$ & $19(3.30)$ & $5.0(0.87)$ & $3.0(0.52)$ & $4(0.69)$ & $7(1.22)$ & $<0.001$ \\
\hline E\% protein & $18.0(17.6,18.4)$ & $17.9(17.5,18.3)$ & $18.2(17.8,18.6)$ & $17.0(16.6,17.4)$ & $16.1(15.7,16.5)$ & $<0.001$ \\
\hline E\% lipids & $39.0(38.3,39.7)$ & $38.5(37.8,39.2)$ & $38.6(37.9,39.3)$ & $38.3(37.6,39.0)$ & $41.0(40.3,41.7)$ & 0.001 \\
\hline E\% carbohydrate & $44.8(44.0,45.6)$ & $45.7(45.0,46.5)$ & $44.9(44.2,45.7)$ & $46.4(45.6,47.2)$ & $45.1(44.3,45.9)$ & 0.336 \\
\hline$E \%$ SFA & $11.6(11.3,11.9)$ & $12.3(11.9,12.6)$ & $12.6(12.3,13.0)$ & $13.2(12.9,13.5)$ & $15.4(15.1,15.7)$ & $<0.001$ \\
\hline E\% MUFA & $15.9(15.5,16.3)$ & $15.7(15.3,16.1)$ & $16.1(15.7,16.4)$ & $15.9(15.5,16.3)$ & $16.8(16.4,17.1)$ & $<0.001$ \\
\hline E\% PUFA & $7.0(6.8,7.2)$ & $6.0(5.8,6.2)$ & $5.4(5.2,5.6)$ & $4.8(4.6,5.0)$ & $4.3(4.1,4.5)$ & $<0.001$ \\
\hline Fiber (g/1000 kcal) & $8.5(8.4,8.6)$ & $8.0(7.9,8.1)$ & $7.7(7.6,7.8)$ & $7.5(7.4,7.6)$ & $6.9(6.8,7.0)$ & $<0.001$ \\
\hline Calcium (mg/1000 kcal) & $425(418,431)$ & $442(436,449)$ & $442(435,449)$ & $446(439,453)$ & $444(437,451)$ & 0.37 \\
\hline Magnesium (mg/1000 kcal) & $141(140,143)$ & $138(137,140)$ & $137(135,138)$ & $135(133,136)$ & $127(126,129)$ & $<0.001$ \\
\hline Vitamin C (mg/1000 kcal) & $47.9(46.5,49.2)$ & $42.6(41.2,44.0)$ & $39.8(38.4,41.1)$ & $36.3(35.0,37.7)$ & $27.7(26.3,29.1)$ & $<0.001$ \\
\hline Vitamin E (mg/1000 kcal) & $3.9(3.9,4.0)$ & $3.7(3.6,3.7)$ & $3.4(3.3,3.5)$ & $3.2(3.1,3.2)$ & $2.9(2.9,3.0)$ & $<0.001$ \\
\hline Vitamin $\mathrm{B}_{6}(\mathrm{mg} / 1000 \mathrm{kcal})$ & $0.87(0.86,0.88)$ & $0.87(0.85,0.88)$ & $0.86(0.85,0.87)$ & $0.81(0.80,0.82)$ & $0.75(0.74,0.76)$ & $<0.001$ \\
\hline Vitamin $B_{2}(\mathrm{mg} / 1000 \mathrm{kcal})$ & $0.81(0.80,0.83)$ & $0.84(0.82,0.85)$ & $0.84(0.82,0.85)$ & $0.83(0.82,0.84)$ & $0.82(0.80,0.83)$ & $<0.001$ \\
\hline $\begin{array}{l}\text { Vitamin } B_{1}(\mathrm{mg} / 1000 \mathrm{kcal}) \\
\text { Food }\end{array}$ & $0.67(0.66,0.68)$ & $0.68(0.67,0.68)$ & $0.67(0.66,0.68)$ & $0.67(0.66,0.68)$ & $0.62(0.62,0.63)$ & $<0.001$ \\
\hline Fruits (g/1000 kcal) & $140(132,148)$ & $109(101,117)$ & $94.0(86.7,102)$ & $76.6(68.6,84.6)$ & $41.3(33.3,49.3)$ & $<0.001$ \\
\hline Vegetables (g/1000 kcal) & $87.6(82.4,92.8)$ & $61.2(56.0,66.4)$ & $48.8(43.6,53.9)$ & $36.9(31.8,42.1)$ & $19.2(14.0,24.4)$ & $<0.001$ \\
\hline Dairy (g/1000 kcal) & $158(149,168)$ & $183(173,192)$ & $190(181,200)$ & $202(193,212)$ & $213(204,234)$ & $<0.001$ \\
\hline Cereals (g/1000 kcal) & $94.2(90.5,98.0)$ & $92.9(89.1,96.7)$ & $88.1(84.3,91.9)$ & $91.1(87.3,94.9)$ & $89.8(86.0,93.6)$ & $<0.001$ \\
\hline Meat (g/1000 kcal) & $32.4(28.9,35.9)$ & $32.2(28.7,35.8)$ & $38.8(35.3,42.3)$ & $37.3(33.8,40.8)$ & $39.8(36.2,43.3)$ & $<0.001$ \\
\hline Legumes ( $\mathrm{g} / 1000 \mathrm{kcal})$ & $12.8(10.8,14.8)$ & $12.6(10.6,14.5)$ & $10.5(8.5,12.4)$ & $9.2(7.2,11.1)$ & $5.7(3.7,7.7)$ & $<0.001$ \\
\hline Fish (g/1000 kcal) & $44.6(41.5,47.8)$ & $29.8(26.7,32.9)$ & $24.3(21.1,27.4)$ & $15.2(12.1,18.3)$ & $8.68(5.54,11.8)$ & $<0.001$ \\
\hline Pastry (g/1000 kcal) & $23.2(20.6,25.8)$ & $20.7(18.1,23.3)$ & $21.1(18.5,23.7)$ & $23.9(21.3,26.5)$ & $28.0(25.4,30.6)$ & 0.002 \\
\hline Cakes, sweets (g/1000 kcal) & $10.2(9.05,11.4)$ & $11.3(10.2,12.5)$ & $11.8(10.6,12.9)$ & $13.3(12.1,14.5)$ & $16.6(15.4,17.8)$ & $<0.001$ \\
\hline
\end{tabular}

Data are shown as means and 95\% confidence interval for continuous variables (KIDMED index score, dietary antioxidant capacity, dietary energy density, energy intake, nutrients, and foods), and proportions for categorical variables (energy underreporting and overreporting).

${ }^{a} p$ Values were obtained by ANOVA, Kruskal-Wallis, and Pearson chi-square for normal continuous, non-normal continuous, and categorical variables, respectively.

SFA, saturated fatty acid; MUFA, monounsaturated fatty acid; PUFA, polyunsaturated fatty acid. 
Table 3. General linear models of the association between diet quality and dietary inflammatory potential measured by the dietary inflammatory index (DII).

\begin{tabular}{|c|c|c|c|c|c|c|}
\hline & $\begin{array}{c}\text { Q1 } \\
(n=578)\end{array}$ & $\begin{array}{c}\text { Q2 } \\
(n=579)\end{array}$ & $\begin{array}{c}\text { Q3 } \\
(n=577)\end{array}$ & $\begin{array}{c}\mathrm{Q} 4 \\
(n=578)\end{array}$ & $\begin{array}{c}\text { Q5 } \\
(n=577)\end{array}$ & $\boldsymbol{p}^{\mathrm{a}}$ \\
\hline \multicolumn{7}{|l|}{$\begin{array}{l}\text { Diet quality measures } \\
\text { Model } 1^{b}\end{array}$} \\
\hline Energy density $(\mathrm{kcal} / \mathrm{g})$ & $0.94(0.91 ; 0.97)$ & $1.02(0.99 ; 1.04)$ & $1.07(1.04 ; 1.09)$ & $1.12(1.09 ; 1.15)$ & $1.20(1.17 ; 1.23)$ & $<0.001$ \\
\hline $\mathrm{TAC}(\mathrm{mmol} / \mathrm{L})$ & $10.0(9.33 ; 10.7)$ & $9.40(8.72 ; 10.0)$ & $8.85(8.17 ; 9.53)$ & $9.27(8.59 ; 9.96)$ & $9.02(8.33 ; 9.72)$ & 0.068 \\
\hline KIDMED index (unit) & $7.85(7.67 ; 8.03)$ & $7.33(7.16 ; 7.51)$ & $7.01(6.86 ; 7.18)$ & $6.91(6.74 ; 7.08)$ & $6.56(6.39 ; 6.74)$ & $<0.001$ \\
\hline \multicolumn{7}{|l|}{ Model $2^{c}$} \\
\hline Energy density (kcal/g) & $0.95(0.92 ; 0.98)$ & $1.02(1.00 ; 1.05)$ & $1.07(1.04 ; 1.09)$ & $1.11(1.09 ; 1.14)$ & $1.19(1.16 ; 1.22)$ & $<0.001$ \\
\hline $\mathrm{TAC}(\mathrm{mmol} / \mathrm{L})$ & $10.2(9.51 ; 10.9)$ & $9.40(8.72 ; 10.0)$ & $8.79(8.11 ; 9.47)$ & $9.21(8.52 ; 9.90)$ & $9.02(8.31 ; 9.72)$ & 0.030 \\
\hline KIDMED index (unit) & $7.77(7.59 ; 7.96)$ & $7.33(7.16 ; 7.50)$ & $7.05(6.88 ; 7.23)$ & $6.93(6.76 ; 7.10)$ & $6.62(6.44 ; 6.80)$ & $<0.001$ \\
\hline \multicolumn{7}{|c|}{$\begin{array}{l}Z \text { scores as diet quality measures } \\
\text { Model } 1^{b}\end{array}$} \\
\hline Energy density (kcal/g) & $-0.37(-0.45 ;-0.29)$ & $-0.15(-0.23 ;-0.07)$ & $-0.01(-0.08 ; 0.06)$ & $0.14(0.06 ; 0.22)$ & $0.36(0.29 ; 0.44)$ & $<0.001$ \\
\hline $\mathrm{TAC}(\mathrm{mmol} / \mathrm{L})$ & $0.07(0.00 ; 0.15)$ & $0.00(-0.06 ; 0.08)$ & $-0.05(-0.12 ; 0.02)$ & $-0.00(-0.08 ; 0.07)$ & $-0.03(-0.11 ; 0.04)$ & 0.068 \\
\hline KIDMED index (unit) & $0.33(0.25 ; 0.42)$ & $0.09(0.01 ; 0.17)$ & $-0.05(-0.13 ; 0.02)$ & $-0.10(-0.18 ;-0.24)$ & $-0.26(-0.35 ;-0.18)$ & $<0.001$ \\
\hline \multicolumn{7}{|l|}{ Model $2^{c}$} \\
\hline Energy density $(\mathrm{kcal} / \mathrm{g})$ & $-0.33(-0.41 ;-0.25)$ & $-0.13(-0.21 ;-0.57)$ & $-0.00(-0.08 ; 0.07)$ & $0.13(0.05 ; 0.20)$ & $0.35(0.27 ; 0.43)$ & $<0.001$ \\
\hline $\mathrm{TAC}(\mathrm{mmol} / \mathrm{L})$ & $0.10(0.02 ; 0.18)$ & $0.00(-0.06 ; 0.08)$ & $-0.05(-0.13 ; 0.01)$ & $-0.01(-0.08 ; 0.06)$ & $-0.03(-0.11 ; 0.04)$ & 0.030 \\
\hline KIDMED index (unit) & $0.30(0.21 ; 0.38)$ & $0.09(0.01 ; 0.17)$ & $-0.03(-0.11 ; 0.04)$ & $-0.09(-0.17 ;-0.01)$ & $-0.24(-0.32 ;-0.15)$ & $<0.001$ \\
\hline
\end{tabular}

Data are shown as means and $95 \%$ confidence interval.

${ }^{a} p$ values were obtained by polynomial contrast.

${ }^{\mathrm{b}}$ Model 1: adjusted for gender and age.

'Model 2: additionally adjusted for maternal education level, community size, region, energy, and energy underreporting. Energy was not included in the energy density analysis.

TAC, total antioxidant capacity.

contrast, dietary energy density ( $\mathrm{kcal} / \mathrm{g})(p<0.001)$ increased across quintiles of the DII. Including caloric beverages in the energy density calculation did not affect this association ( $p$ linear trend $<0.001$ ).

We determined the degree of agreement between the three diet quality measures. Cohen's $\kappa$ showed poor strength of agreement between the KIDMED index and total dietary antioxidant capacity $(\kappa=0.011$, $p=0.421)$, the KIDMED index and energy density $(\kappa=-0.080, p=0.001)$, and total dietary antioxidant capacity and energy density $(\kappa=-0.083, p=0.001)$.

No significant interaction between DII and energy overreporting and underreporting was found.

Sensitivity analysis was used to assess the robustness of our results under various scenarios. Multivariate linear regression analysis stratified by gender and age groups revealed no meaningful differences when stratified by gender and age [children (6-11 years), adolescents (12-17 years), and young adults (18-24 years)] (Supplementary Table 1).

\section{Discussion}

In this cross-sectional study in Spanish youth, higher diet quality was associated with increased dietary antiinflammatory potential in three conceptually different measures validated in the study population. The strength of agreement between the selected indices was poor and correlations between measures were poor to fair; the one indicator that scored highly on all three was greater dietary anti-inflammatory potential. This finding makes an important contribution to the debate about defining and measuring diet quality.

Diet plays a pivotal role in inflammatory status because foods and nutrients modulate inflammatory processes [1-3]. The DII was created to measure the inflammatory potential of diets [7], based on a systematic review of literature reporting the effect of diet on inflammation. Thus, the DII has been used to determine the inflammatory profile of various diets [16,17,30-32]. In 2009-2010 data from the US National Health and Nutrition Examination Survey, a macrobiotic diet showed a significantly higher anti-inflammatory potential compared with the average US diet [30]; similarly, the DII indicated that the macrobiotic diet had more anti-inflammatory potential than the average US diet (mean DII average score of -1.88 and 1.00 , respectively). Assessment of the dietary inflammatory potential of fast food, Mediterranean, and macrobiotic diet plans revealed a strong pro-inflammatory potential for the fast food diet; the opposite was true for the Mediterranean and macrobiotic diets [31]. Furthermore, adopting a vegan, vegetarian, or pescovegetarian diet was linked to a higher dietary anti-inflammatory potential, compared to adopting a semi-vegetarian diet after 2 months of dietary intervention in overweight and obese study participants [16].

Nutritional epidemiology offers several approaches to assessing overall diet quality [18]. Numerous dietary indices have been developed in the attempt to measure overall diet quality [33-35]. A recently published study [17] investigated the association of three diet quality measures (Healthy Eating Index 2010, the Alternative 
Healthy Eating Index, and the Dietary Approaches to Stop Hypertension Index) with dietary inflammatory potential as measured by the DII. In young adults, they found an increase in the anti-inflammatory potential associated with higher scores, indicating a healthier diet, on all three instruments in young adults [17].

The present study used three conceptually different measures of diet quality - energy density, antioxidant capacity, and the KIDMED index - to test our hypothesis that higher diet quality is related to a more favorable dietary inflammatory potential in young people. These diet quality indicators capture different dietary dimensions and have been directly associated with healthy food consumption in the present population $[22,36]$.

In both adult and child populations, diets with low energy density are characterized by a high consumption of healthy foods, such as vegetables and fruits, reflecting a high diet quality [36,37]. Total dietary antioxidant capacity is based on the rationale that a diet rich in foods with high antioxidant potential will help to counterattack the harmful effects of free radicals in body tissues [38]. Adherence to the Mediterranean diet, as measured by the KIDMED index, reflects a priori selection of healthy foods and food habits. Notably, our results showed a poor agreement between these indices, indicating different patterns of food consumption within a healthy diet according to the measure used. However, a common characteristic of all three indices was the decrease in dietary inflammatory potential as diet quality scores increased. The lower effect size of the total dietary antioxidant potential compared with energy density and KIDMED scores was somewhat surprising; the amount of pro-inflammatory nutrients in foods which also exhibit antioxidant properties, such as meat and sausages, offers one potential explanation.

Several studies have shown that energy density is positively associated with energy intake in adult and younger populations $[36,39]$. It was therefore somewhat surprising that energy density increased with the inflammatory potential of the diet, with a concomitant decrease in total energy intake, in the present study. However, this does not necessarily imply that individuals following a high energy density diet will also have higher energy intakes compared with those adhering to a low energy density diet. Numerous food combinations show differences in total energy content for a given unit of energy density. Further studies are needed to verify whether lower energy intake despite higher energy density is a characteristic of increased inflammatory dietary potential in other populations.

Consistent with results from a previous study by SerraMajem and colleagues [21], KIDMED scores were higher, indicating a healthier diet, in large cities than in small cities; the DII scores showed the same trend. There is emerging evidence that large urbanized cities have better access to healthy and affordable food [40]. In contrast, access to affordable healthy food can be challenging in rural areas with lower population density [40].

A limitation of this study is the cross-sectional study design, which precludes drawing causal relationships. Furthermore, $24 \mathrm{~h}$ recalls have inherent limitations in individual dietary assessment, owing to daily variations in food intake. However, the sample size of 2599 participants was large enough to characterize group intakes. Energy misreporting is an inherent bias in dietary assessment data. Although no interaction was found between the DII and energy misreporting, we adjusted the final data analysis for this potential confounder in order to reduce measurement error. The study also has several important strengths, including a nationwide population-based sample and interviewer-guided completion of questionnaires.

In conclusion, good diet quality, indicated by higher KIDMED index scores, higher total dietary antioxidant capacity, and lower energy density, is positively associated with dietary anti-inflammatory potential as measured by the DII in the Spanish population aged 6-24 years.

\section{Acknowledgements}

We appreciate the English revision by Elaine M. Lilly, PhD. This work was supported by grants from the Spanish Ministry of Health [RED: Alimentación saludable en la prevención primaria de enfermedades crónicas: la Red Predimed, one of the Redes Temáticas de Investigación Cooperativa Sanitaria (RETICs)] Fundación Dieta Mediterránea, Spain; and Kellogg's España SA and Kellogg's Company, Battle Creek, USA, via the Fundación Universitaria de Las Palmas de Gran Canaria and the Fundación para la Investigación Nutricional, and by the King Abdullah scholarship program [2014, ID 2631], Instituto de Salud Carlos III FEDER (CB06/02/0029), AGAUR (2014 SGR 240). The CIBERESP and the CIBEROBN and the RETIC Predimed are initiatives of the Instituto de Salud Carlos III, Madrid, Spain. The funders had no role in study design, data collection and analysis, decision to publish, or preparation of the manuscript.

\section{Disclosure statement}

No potential conflict of interest was reported by the authors.

\section{Funding}

This work was supported by the Spanish Ministry of Health; Fundación Dieta Mediterránea, Spain; Kellogg's España SA and Kellogg's Company; Fundación Universitaria de Las Palmas de Gran Canaria; Fundación para la Investigación Nutricional; King Abdullah scholarship program [2014, ID 2631]; Instituto de Salud Carlos III FEDER [CB06/02/0029]; and AGAUR [2014 SGR 240]. 


\section{References}

[1] King DE, Egan BM, Geesey ME. Relation of dietary fat and fiber to elevation of C-reactive protein. Am J Cardiol. 2003;92(11):1335-1339.

[2] Esposito K, Marfella R, Ciotola M, et al. Effect of a mediterranean-style diet on endothelial dysfunction and markers of vascular inflammation in the metabolic syndrome: a randomized trial. JAMA. 2004;292(12):1440-1446.

[3] Lopez-Garcia E, Schulze MB, Meigs JB, et al. Consumption of trans fatty acids is related to plasma biomarkers of inflammation and endothelial dysfunction. J Nutr [Internet]. 2005 Mar 1 [cited 2016 Aug 30];135(3):562-566. Available from: http://jn.nutrition. org/cgi/content/long/135/3/562

[4] Ridker PM, Rifai N, Rose L, et al. Comparison of C-reactive protein and low-density lipoprotein cholesterol levels in the prediction of first cardiovascular events. N Engl J Med [Internet]. 2002;347(20):15571565. Available from: http://www.ncbi.nlm.nih.gov/ pubmed/12432042

[5] Keibel A, Singh V, Sharma MC. Inflammation, microenvironment, and the immune system in cancer progression. Curr Pharm Des. 2009;15(17):1949-1955.

[6] Berenson GS. Cardiovascular risk begins in childhood. Am J Prev Med [Internet]. 2009;37(1):S1-2. Available from: http://linkinghub.elsevier.com/retrieve/pii/ S0749379709002591.

[7] Shivappa N, Steck SE, Hurley TG, et al. Designing and developing a literature-derived, population-based dietary inflammatory index. Public Health Nutr [Internet]. 2014;17(8):1689-1696. Available from: http://www.jour nals.cambridge.org/abstract_S1368980013002115

[8] Shivappa N, Steck SE, Hurley TG, et al. A populationbased dietary inflammatory index predicts levels of C-reactive protein in the Seasonal Variation of Blood Cholesterol Study (SEASONS). Public Health Nutr [Internet]. 2014;17(8):1825-1833. Available from: http://www.ncbi.nlm.nih.gov/pubmed/24107546

[9] Wirth MD, Burch J, Shivappa N, et al. Association of a dietary inflammatory index with inflammatory indices and metabolic syndrome among police officers. J Occup Environ Med [Internet]. 2014;56(9):986-989. Available from: http://www.ncbi.nlm.nih.gov/pubmed/25046320

[10] Shivappa N, Hébert JR, Rietzschel ER, et al. Associations between dietary inflammatory index and inflammatory markers in the Asklepios Study. Br J Nutr [Internet]. 2015;(Dii):1-7. Available from: http://www.journals.cam bridge.org/abstract_S000711451400395X

[11] Tabung FK, Steck SE, Zhang J, et al. Construct validation of the dietary inflammatory index among postmenopausal women. Ann Epidemiol [Internet]. 2015;25 (6):398-405. Available from: http://www.sciencedirect. com/science/article/pii/S1047279715001192

[12] Shivappa N, Bosetti C, Zucchetto A, et al. Association between dietary inflammatory index and prostate cancer among Italian men. Br J Nutr [Internet]. 2014;1-6. Available from: http://www.ncbi.nlm.nih.gov/pubmed/ 25400225

[13] Wood LG, Shivappa N, Berthon BS, et al. Dietary inflammatory index is related to asthma risk, lung function and systemic inflammation in asthma. Clin Exp Allergy. 2015;45(1):177-183.

[14] Garcia-Arellano A, Ramallal R, Ruiz-Canela M, et al. Dietary inflammatory index and incidence of cardiovascular disease in the PREDIMED Study. Nutrients [Internet]. 2015;7(6):4124-4138. Available from: http:// www.pubmedcentral.nih.gov/articlerender.fcgi?artid= 4488776\&tool=pmcentrez\&rendertype $=$ abstract

[15] Ruiz-Canela M, Zazpe I, Shivappa N, et al. Dietary inflammatory index and anthropometric measures of obesity in a population sample at high cardiovascular risk from the PREDIMED (PREvención con DIeta MEDiterránea) trial. Br J Nutr [Internet]. 2015;1-12. Available from: http:// www.ncbi.nlm.nih.gov/pubmed/25720588

[16] Turner-McGrievy GM, Wirth MD, Shivappa N, et al. Randomization to plant-based dietary approaches leads to larger short-term improvements in dietary inflammatory index scores and macronutrient intake compared with diets that contain meat. Nutr Res. 2015;35(2):97-106.

[17] Wirth MD, Hébert JR, Shivappa N, et al. Anti-inflammatory dietary inflammatory index scores are associated with healthier scores on other dietary indices. Nutr Res. 2016;36(3):214-219.

[18] Alkerwi A. Diet quality concept. Nutrition. 2014;30:613-618.

[19] Serra Majem L, Ribas Barba L, Aranceta Bartrina J, et al. Obesidad infantil y juvenil en España. Resultados del Estudio enKid (1998-2000). Med Clin (Barc). 2003;121 (19):725-732.

[20] Liu K, Stamler J, Dyer A, et al. Statistical methods to assess and minimize the role of intra-individual variability in obscuring the relationship between dietary lipids and serum cholesterol. J Chronic Dis [Internet]. 1978 [cited 2017 Jan 31];31(6-7):399-418. Available from: http://www.ncbi.nlm.nih.gov/pubmed/711832

[21] Serra-Majem L, Ribas L, Ngo J, et al. Food, youth and the Mediterranean diet in Spain. Development of KIDMED, Mediterranean diet quality index in children and adolescents. Public Heal Nutr [Internet]. 2004;7(7):931-935. Available from: http://journals.cambridge.org/download. php? file=/PHN/PHN7_07/S136898000400117Xa. pdf\&code $=43 f 4492 \mathrm{ef} 126 \mathrm{f} 3513 \mathrm{e} 3 \mathrm{ad} 43 \mathrm{a} 422129 \mathrm{~d} 3$

[22] Serra-Majem L, Ribas L, García A, et al. Nutrient adequacy and Mediterranean diet in Spanish school children and adolescents. Eur J Clin Nutr [Internet]. 2003;57(Suppl 1):S35-9. Available from: http://www.nat ure.com/ejcn/journal/v57/n1s/pdf/1601812a.pdf

[23] Ledikwe JH, Blanck HM, Khan LK, et al. Dietary energy density determined by eight calculation methods in a nationally representative United States population. J Nutr. 2005;135(2):273-278.

[24] Carlsen MH, Halvorsen BL, Holte $\mathrm{K}$, et al. The total antioxidant content of more than 3100 foods, beverages, spices, herbs and supplements used worldwide. Nutr J [Internet]. 2010;9:3. Available from: http://www.pub medcentral.nih.gov/articlerender.fcgi? artid= 2841576\&tool $=$ pmcentrez\&rendertype $=$ abstract

[25] Schofield WN. Predicting basal metabolic rate, new standards and review of previous work. Hum Nutr Clin Nutr. 1985;39(Suppl 1):5-41. 
[26] Goldberg G, Black A, Jebb S, et al. Critical evaluation of energy intake data using fundamental principles of energy physiology: 1. Derivation of cut-off limits to identify under-recording. Eur J Clin Nutr. 1991;45(12):569-581.

[27] Black AE. Critical evaluation of energy intake using the Goldberg cut-off for energy intake: basalmetabolic rate. A practical guide to its calculation, use and limitations. Int J Obes. 2000;24(9):1119-1130.

[28] Nelson M, Black AE, Morris JA, et al. Between- and within-subject variation in nutrient intake from infancy to old age: estimating the number of days required to rank dietary intakes with desired precision. Am J Clin Nutr. 1989;50(1):155-167.

[29] Torun B, Davies PS, Livingstone MB, et al. Energy requirements and dietary energy recommendations for children and adolescents 1 to 18 years old. Eur J Clin Nutr. 1996;50(Suppl 1):S37-80; discussion S80-1.

[30] Harmon BE, Carter M, Hurley TG, et al. Nutrient composition and anti-inflammatory potential of a prescribed macrobiotic diet. Nutr Cancer. 2015;67(6):933-940.

[31] Steck S, Nitin shivappa, Tabung F, Harmon B, Wirth MD, Hurley $\mathrm{T}$, et al.The Dietary Inflammatory Index: The Dietary Inflammatory Index: A New Tool for Assessing Diet Quality Based on Inflammatory Potential. The Digest. 2014;49(3):1-9.

[32] Hodge AM, Bassett JK, Shivappa N, et al. Dietary inflammatory index, Mediterranean diet score, and lung cancer: a prospective study. Cancer Causes Control [Internet]. 2016 Jul 13 [cited 2016 Aug 30];27 (7):907-917. Available from: http://link.springer.com/ 10.1007/s10552-016-0770-1

[33] Haines PS, Siega-Riz AM, Popkin BM. The diet quality index revised: a measurement instrument for populations. J Am Diet Assoc. 1999;99(6):697-704.
[34] Panagiotakos DB, Pitsavos C, Stefanadis C. Dietary patterns: a Mediterranean diet score and its relation to clinical and biological markers of cardiovascular disease risk. Nutr Metab Cardiovasc Dis. 2006;16 (8):559-568.

[35] Marshall S, Burrows T, Collins CE. Systematic review of diet quality indices and their associations with healthrelated outcomes in children and adolescents. J Hum Nutr Diet. 2014;1-22. Available from: http://www.ncbi. nlm.nih.gov/pubmed/24524271

[36] Schröder H, Mendez MA, Gomez SF, et al. Energy density, diet quality, and central body fat in a nationwide survey of young Spaniards. Nutrition. 2013;29(1112):1350-1355.

[37] Ledikwe JH, Blanck HM, Khan LK, et al. Low-energydensity diets are associated with high diet quality in adults in the United States. J Am Diet Assoc. 2006;106 (8):1172-1180.

[38] Puchau B, Zulet MA, de Echávarri AG, et al. Dietary total antioxidant capacity: a novel indicator of diet quality in healthy young adults. J Am Coll Nutr. 2009 Dec;28(6):648-656. Available from: http://www.ncbi. nlm.nih.gov/pubmed/20516264

[39] Schröder H, Covas M, Elosua R, et al. Diet quality and lifestyle associated with free selected low-energy density diets in a representative Spanish population. Eur J Clin Nutr. 2008 Oct 11;62(10):1194-1200. Available from: http://www.ncbi.nlm.nih.gov/ pubmed/17622256

[40] Pinard CA, Shanks CB, Harden SM, et al. An integrative literature review of small food store research across urban and rural communities in the U.S. Prev Med Rep. 2016;3:324-332. Available from: http://www.scien cedirect.com/science/article/pii/S2211335516300092 